\title{
EQUILIBRIUM STELLAR SYSTEMS WITH SPINDLE SINGULARITIES
}

\author{
Stuart L. Shapiro ${ }^{1}$ and SaUl A. Teukolsky ${ }^{1}$ \\ Center for Radiophysics and Space Research, Cornell University \\ Received 1991 July 19; accepted 1991 September 26
}

\begin{abstract}
We construct equilibrium sequences of axisymmetric Newtonian clusters that tend toward singular states. The distribution functions are chosen to be of the form $f=f\left(E, J_{z}\right)$. The numerical method then determines the density and gravitational potential self-consistently to satisfy Poisson's equation. For the prolate models, spindle singularities arise from the depletion of angular momentum near the symmetry axis. While the resulting density enhancement is confined to the region near the axis, the influence of the spindle extends much further out through its tidal gravitational field. Centrally condensed prolate clusters may contain strongfield regions even though the spindle mass is small and the mean cluster eccentricity is not extreme.

While the calculations performed here are entirely Newtonian, the issue of singularities is an important topic in general relativity. Equilibrium solutions for relativistic star clusters can provide a testing ground for exploring this issue. The methods used in this paper for building nonspherical clusters can be extended to relativistic systems.
\end{abstract}

Subject headings: celestial mechanics, stellar dynamics - galaxies: kinematics and dynamics globular clusters: general — relativity

\section{INTRODUCTION}

\subsection{Background}

There is considerable interest in the structure of nonspherical stellar systems in dynamical equilibrium. Physically, such systems exist as galaxies and globular star clusters. Mathematically, they are self-consistent solutions to the Vlasov equation for the stellar distribution function $f$ coupled to Poisson's equation for the gravitational potential $\Phi$. Finding such solutions is difficult when the configuration is not spherical and the number of nontrivial phase-space degrees of freedom is large. (For a general review and discussion, see Fridman \& Polyachenko 1984 or Binney \& Tremaine 1987.)

In this paper we are interested in sequences of nonspherical stellar systems that approach a singular state. Examples of singular systems include axisymmetric clusters with prolate spindles along their axis, or with oblate pancakes along their equator. Prolate spindles are especially interesting: their gravitational fields are more strongly singular than those of pancakes, and they cannot arise in equilibrium fluids, but only in collisionless systems.

Most of the effort in constructing nonspherical clusters has been devoted to oblate models for elliptical galaxies. The possibility that some observed features might be explained by prolate structures has stimulated the construction of a few prolate models (see Lake 1981a, b for examples and references). As clusters become increasingly prolate, their gravitational fields become increasingly singular. Tremaine \& De Zeeuw (1987) have considered the limiting case of strictly onedimensional needles. However, a systematic procedure for studying the approach to singularity of multidimensional systems has not been previously presented. In part, this is because highly aspherical configurations are computationally challenging.

The simplest equilibrium solutions to the Vlasov equation

\footnotetext{
${ }^{1}$ Departments of Astronomy and Physics, Space Sciences Building, Cornell University, Ithaca, NY 14853.
}

with axisymmetry are of the form $f=f\left(E, J_{z}\right)$, where $E$ is the energy and $J_{z}$ is the angular momentum about the symmetry axis, both per unit mass. By suitable choice of the distribution in $J_{z}$, one can construct models that are either prolate or oblate. A depletion in the $J_{z}$-distribution produces a prolate configuration, while an enhancement produces an oblate one. The easiest way to implement this procedure is to write

$$
f\left(E, J_{z}\right)=g(E) h\left(J_{z}\right)
$$

and vary $h$ appropriately. When $h$ is constant, we get spherical models with isotropic velocity distributions. In this paper, we construct generalized polytropes by choosing $g$ to be the same function of $E$ that gives rise to ordinary polytropes in the spherical case. By varying the polytropic index, we can study systems that range from nearly homogeneous to highly centrally condensed. With a self-consistent solution in hand, we can calculate its density profile, velocity distribution, and gravitational tidal field strength. As $h$ is varied, we can build entire sequences of nonspherical equilibria and study their approach to singular states.

While the calculations in this paper are strictly Newtonian, they also address the important issue of singularities in general relativity theory. This issue is discussed in the next subsection. The remainder of the paper is entirely Newtonian and is organized as follows. In $\S 2$ we present the key equations and diagnostic probes that describe equilibrium clusters. In $\S 3$ we outline the numerical method. Section 4 summarizes some analytic results that are helpful in assessing the numerical models. Section 5 presents the numerical results, while the conclusions are given in $\S 6$.

\subsection{Relevance for General Relativity}

The well-known Newtonian instability discussed by Lin, Mestel, \& Shu (1965) shows that the collapse of a nonrotating homogeneous spheroid of collisionless matter can lead to the formation of singularities. If the spheroid is slightly oblate initially, the configuration collapses to a pancake, while if the spheroid is slightly prolate, it collapses to a spindle. Although 
in both cases the density becomes infinite, the formation of a spindle during prolate collapse is particularly significant. The gravitational potential, gravitational force, tidal force, and kinetic and potential energies all blow up to infinity. Prolate evolution is forced to terminate at the singular spindle state. For oblate evolution, the matter simply passes through the pancake state, but then becomes prolate and also evolves to a spindle singularity.

Can such a singular spindle form during relativistic collapse? And if so, will the singularity always be hidden inside a black hole? The cosmic censorship hypothesis (Penrose 1969) states that all singularities formed during collapse will be inside black holes, clothed by event horizons, and hence never visible from the outside (no naked singularities). The formation of a naked singularity would be a disaster for general relativity because the theory could not predict the future evolution. The hoop conjecture (Thorne 1972) states that a black hole will form if and only if collapsing matter becomes sufficiently compact in all directions. It is difficult to reconcile the hoop conjecture and cosmic censorship: a sufficiently long spindle could not form a black hole, so any singularity that formed would be naked.

Recent advances in numerical relativity now make it possible to address this dilemma by computer calculation. In particular, we have carried out numerical simulations of collisionless spheroid collapse in full general relativity (Shapiro \& Teukolsky 1991). Our numerical results lend support to the hoop conjecture and provide evidence that naked singularities can in fact form in general relativity. One limitation of such simulations is that the integrations themselves are forced to terminate as soon as a singularity appears. Thus, while there was no evidence of a black hole when our simulations ended (no "apparent horizon"), there is no guarantee that outgoing light rays would have continued outward forever (no "event horizon"). Hence our conclusion that naked singularities can form is not yet watertight.

How can we probe the nature of singularities without the ambiguities of unpredictable future dynamical evolution? One way is to explore them in equilibrium configurations. (Apparent and event horizons coincide for stationary configurations.) We propose to examine prolate spindle singularities in fully relativistic equilibrium clusters. As a first step, we examine in this paper their Newtonian counterparts. This preliminary study will provide not only valuable physical insight, but also a numerical approach that will generalize to the relativistic case. With this future generalization in mind, we restrict our attention here to the simplest distribution functions that can give rise to nonspherical equilibria, functions of $E$ and $J_{z}$ alone. While distribution functions depending on three or more integrals of the motion can be constructed to give rise to spindles in Newtonian gravitation, there is no obvious way to generalize them to the relativistic case.

The singularity that forms during nonspherical Lin-MestelShu collapse in Newtonian theory can be prevented by any slight initial inhomogeneity or velocity dispersion (see Horst 1982 for a formal proof; see Shapiro \& Teukolsky 1987 for a numerical demonstration). However, we know from countless numerical simulations that small perturbations are not at all sufficient to prevent singularity formation during relativistic collapse (see Rendall 1991 for a formal proof for spherical collisionless collapse). The unanswered question is whether or not the singularity that forms during collapse can ever be naked. We will be able to assess the conditions leading to the formation of naked singularities by studying equilibrium configurations.

\section{KEY EQUATIONS}

Any phase-space distribution function $f\left(E, J_{z}\right)$ is an equilibrium solution of the Vlasov equation in axisymmetry. The energy per unit mass $E$ is given by

$$
E=\frac{1}{2 m^{2}}\left(p_{\perp}^{2}+p_{\phi}^{2}\right)+\Phi(r),
$$

where $m$ is the particle mass, $p_{\phi}$ is the momentum about the symmetry axis, $p_{\perp}$ is the momentum perpendicular to $p_{\phi}$, and $\Phi$ is the gravitational potential. We adopt spherical polar coordinates $(r, \theta, \phi)$ and choose the $z$-direction along the axis. Instead of $\theta$, we use

$$
x=\cos \theta,
$$

so that $\Phi=\Phi(r, x)$. The angular momentum per unit mass about the $z$-axis is

$$
J_{z}=r\left(1-x^{2}\right)^{1 / 2} p_{\phi}
$$

The stellar density $\rho$ is given by

$$
\rho=m \int f d^{3} p,
$$

and must be determined self-consistently with the potential according to Poisson's equation.

We shall only consider bound systems of finite extent, for which $E$ has a maximum value $E_{\max }<0$. For such systems, equation (2.4) becomes

$$
\begin{aligned}
\rho(r, x)=4 \pi m \int_{0}^{\left[2\left(E_{\max }-\Phi\right)\right]^{1 / 2}} d p_{\perp} p_{\perp} \\
\quad \times \int_{0}^{\left[2\left(E_{\max }-\Phi\right)-p_{\perp}^{2} / m^{2}\right]^{1 / 2}} d p_{\phi} f\left(E, J_{z}\right),
\end{aligned}
$$

where $E$ and $J_{z}$ are defined in equations (2.1) and (2.3). The solution of Poisson's equation can be written

$$
\begin{aligned}
\Phi(r, x)=-4 \pi G \sum_{l=0}^{\infty} P_{2 l}(x) \int_{0}^{\infty} & d r^{\prime} r^{\prime 2} \frac{r_{<}^{2 l}}{r_{>}^{2 l+1}} \\
& \times \int_{0}^{1} d x^{\prime} P_{2 l}\left(x^{\prime}\right) \rho\left(r^{\prime}, x^{\prime}\right),
\end{aligned}
$$

where $P_{l}(x)$ is a Legendre polynomial and where we have assumed reflection symmetry about the equatorial plane. Here $r_{<}$is the lesser of $r$ and $r^{\prime}$, and similarly for $r_{>}$. The total mass $M$ of the system is

$$
M=4 \pi \int_{0}^{\infty} d r r^{2} \int_{0}^{1} d x \rho(r, x) .
$$

It will prove useful to expand $\rho$ and $\Phi$ in Legendre polynomials:

$$
\begin{aligned}
& \rho(r, x)=\sum_{l=0}^{\infty} \rho_{2 l}(r) P_{2 l}(x), \\
& \Phi(r, x)=\sum_{l=0}^{\infty} \Phi_{2 l}(r) P_{2 l}(x),
\end{aligned}
$$

where

$$
\rho_{2 l}(r)=(4 l+1) \int_{0}^{1} d x \rho(r, x) P_{2 l}(x),
$$


and similarly for $\Phi_{2 l}(r)$. In a numerical solution, we truncate these expansions at some large, finite value $L$ of $l$. Adopting such a spectral representation of the angular dependence, we get more rapid convergence of the solution with increasing $L$ compared with, for example, a standard finite-difference approach (see, e.g., Gottlieb \& Orszag 1977 for a discussion).

The entire problem can be greatly simplified by using the scale freedom to remove $G, m, M$, and $E_{\max }$ from all the equations. This is accomplished by defining nondimensional variables as follows:

$$
\begin{gathered}
\tilde{E}=\frac{E}{\left|E_{\max }\right|}, \quad \tilde{J}_{z}=\frac{J_{z}}{G M /\left|E_{\max }\right|^{1 / 2}}, \quad \tilde{\Phi}=\frac{\Phi}{\left|E_{\max }\right|} \\
\tilde{p}=\frac{p}{m\left|E_{\max }\right|^{1 / 2}}, \quad \tilde{r}=\frac{r}{G M /\left|E_{\max }\right|}, \quad \tilde{\rho}=\frac{\rho}{M\left[G M /\left|E_{\max }\right|\right]^{-3}}, \\
\tilde{f}=\frac{f}{(M / m)\left[G M m /\left|E_{\max }\right|^{1 / 2}\right]^{-3}} .
\end{gathered}
$$

We now drop all the tildes, so that henceforth all equations are written in terms of these nondimensional quantities. This is equivalent to setting $G=1=m=M=-E_{\max }$ in all of our equations. In particular, equations (2.5)-(2.7) become

$$
\begin{aligned}
\rho(r, x)= & 4 \pi \int_{0}^{|2(1+\Phi)|^{1 / 2}} d p_{\perp} p_{\perp} \int_{0}^{\left|2(1+\Phi)+p_{\perp} 2\right| 1 / 2} \\
& \times d p_{\phi} f\left[\frac{1}{2}\left(p_{\perp}^{2}+p_{\phi}^{2}\right)+\Phi, r\left(1-x^{2}\right)^{1 / 2} p_{\phi}\right] \\
\Phi_{2 l}(r)= & \frac{-4 \pi}{4 l+1} \int_{0}^{\infty} d r^{\prime} r^{2} \frac{r_{<}^{2 l}}{r_{>}^{2 l+1}} \rho_{2 l}\left(r^{\prime}\right) \\
1= & 4 \pi \int_{0}^{\infty} d r r^{2} \rho_{0}(r) .
\end{aligned}
$$

These are the fundamental equations that must be solved selfconsistently. We discuss our numerical method for solving these equations by iteration in $\S 3$.

\subsection{Diagnostics}

Once the fundamental equations (2.11)-(2.13) have been solved, we can probe the structure of the cluster by calculating a number of physically important diagnostics. First, the mean squared velocities are given by

$$
\begin{aligned}
& v_{\perp}^{2}(r, x)=\frac{1}{\rho} \int d p_{\perp} p_{\perp}^{3} \int d p_{\phi} f \\
& v_{\phi}^{2}(r, x)=\frac{1}{\rho} \int d p_{\perp} p_{\perp} \int d p_{\phi} p_{\phi}^{2} f
\end{aligned}
$$

where the limits of integration are the same as in equation (2.11). For any $f=f\left(E, J_{z}\right)$, the velocity vector is isotropically distributed in a plane orthogonal to the $\phi$-direction and so no information is gained by decomposing $v_{\perp}^{2}$ into two orthogonal components. The structure of the cluster does not depend on what fraction of the particles are moving in the $+\phi$-direction versus the $-\phi$-direction. If equal numbers are moving in each direction, the net angular momentum of the cluster is in fact zero. We will let $\chi$ denote the fraction of the particles moving in the $+\phi$-direction, where $0 \leq \chi \leq 1$.

The net rotational energy of the cluster is

$$
T_{\text {rot }}=2 \pi(2 \chi-1)^{2} \int_{0}^{\infty} d r r^{2} \int_{0}^{1} d x \rho(r, x) v_{\phi}^{2}(r, x) .
$$

The total kinetic energy is

$$
T=2 \pi \int_{0}^{\infty} d r r^{2} \int_{0}^{1} d x \rho(r, x)\left[v_{\phi}^{2}(r, x)+v_{\perp}^{2}(r, x)\right] .
$$

The total potential energy is

$$
\begin{aligned}
W & =2 \pi \int_{0}^{\infty} d r r^{2} \int_{0}^{1} d x \rho(r, x) \Phi(r, x) \\
& =2 \pi \sum_{l=0}^{\infty} \frac{1}{4 l+1} \int_{0}^{\infty} d r r^{2} \rho_{2 l}(r) \Phi_{2 l}(r) .
\end{aligned}
$$

Hence the total energy is

$$
E_{\text {tot }}=T+W=W / 2,
$$

where the last equality follows from the virial theorem

$$
2 T+W=0 \text {. }
$$

When expressed nondimensionally, these energies are all in units of $M\left|E_{\max }\right|$. For a spherical polytrope of radius $R$ and polytropic index $n,\left|E_{\max }\right|=G M / R$ and

$$
E_{\mathrm{tot}}=-\frac{3}{2(5-n)}
$$

in our nondimensional units.

We use the quadrupole moments of the mass distribution to define a global eccentricity $e$ of the cluster. The principal moments of inertia are

$$
\begin{aligned}
I_{x x}=I_{y y} & =\int \rho\left(y^{2}+z^{2}\right) d^{3} x, \\
I_{z z} & =\int \rho\left(x^{2}+y^{2}\right) d^{3} x .
\end{aligned}
$$

Substituting from equation (2.8), we find

$$
\begin{aligned}
& I_{x x}=\frac{4 \pi}{3} \int_{0}^{\infty} d r r^{4}\left[2 \rho_{0}(r)+\frac{1}{5} \rho_{2}(r)\right], \\
& I_{z z}=\frac{8 \pi}{3} \int_{0}^{\infty} d r r^{4}\left[\rho_{0}(r)-\frac{1}{5} \rho_{2}(r)\right] .
\end{aligned}
$$

We then define the eccentricity by

$$
e^{2}=\left\{\begin{array}{lll}
\frac{I_{x x}-I_{z z}}{I_{x x}-I_{z z} / 2}, & I_{x x}>I_{z z} & \text { (prolate) }, \\
\frac{2\left(I_{z z}-I_{x x}\right)}{I_{z z}}, & I_{x x}<I_{z z} & \text { (oblate) } .
\end{array}\right.
$$

For a homogeneous spheroid with equatorial semiaxis $a$ and polar semiaxis $c$, this definition reduces to the standard expression for eccentricity,

$$
e^{2}= \begin{cases}1-\frac{a^{2}}{c^{2}} & \text { (prolate) } \\ 1-\frac{c^{2}}{a^{2}} & \text { (oblate) }\end{cases}
$$

Centrally condensed clusters with very nonspherical structures in the core may still yield small values of the eccentricity (2.23). For such systems the nonspherical structures show up in diagnostics that depend only on local properties, such as density contour plots, and not on global averages that weight the outer regions. 
Consider now how we might diagnose the presence of a singularity in a cluster. The gravitational force at a point is not a satisfactory diagnostic because its influence on a particle there can be removed simply by comoving with the particle in free fall. A singular tidal force, by contrast, causes the relative acceleration of neighboring particles to become infinite. There is no global reference frame that can simultaneously comove with all the particles in free fall. Hence the blowup of the tidal force is a better criterion for a singularity in the gravitational field. For a nonspherical star cluster, such a region can occur both inside and outside the cluster.

Another criterion for a singularity is whether trajectories are forced to terminate because of infinities arising in the equations of motion. Points where this occurs then define the singular region.

These two criteria for singularity are not always equivalent. We shall see an example of this for oblate pancakes in $\S 4.1$.

As a measure of the gravitational tidal field, consider the tidal tensor

$$
\Phi_{i j} \equiv \frac{\partial^{2} \Phi}{\partial x_{i} \partial x_{j}}
$$

in Cartesian coordinates. From this quantity, we construct a simple scalar function:

$$
I=8 \Phi_{i j} \Phi_{i j}
$$

where summation over repeated indices is implied. ${ }^{2}$ Blowing up of $I$ signals the presence of a singularity. In axisymmetry,

$$
\begin{aligned}
I= & 8\left[\Phi_{, r r}^{2}+2\left(\frac{1}{r^{2}} \Phi_{, r x}-\frac{1}{r^{2}} \Phi_{, x}\right)^{2}\left(1-x^{2}\right)\right. \\
& +\left(\frac{1-x^{2}}{r^{2}} \Phi_{, x x}+\frac{1}{r} \Phi_{, r}-\frac{x}{r^{2}} \Phi_{, x}\right)^{2} \\
& \left.+\left(\frac{1}{r^{2}} \Phi_{, r}-\frac{x}{r^{2}} \Phi_{, x}\right)^{2}\right],
\end{aligned}
$$

where a comma denotes a partial derivative.

\subsection{Adopted Distribution Function}

For the generalized polytropes that we consider, we choose $g$ in equation (1.1) to be of the form

$$
g(E)= \begin{cases}K[-(E+1)]^{n-3 / 2}, & E<-1, \\ 0, & E \geq-1 .\end{cases}
$$

Here $n$ is the polytropic index, which ranges over the allowed values $\frac{1}{2}$ to 5 . The factor $K$ is an overall normalization constant whose value is determined by fixing the total mass. For the angular momentum function $h$ in equation (1.1), we choose a Gaussian,

$$
h\left(J_{z}\right)=\frac{1}{\pi^{1 / 2} J_{0}} \exp \left(\frac{\mp J_{z}^{2}}{J_{0}^{2}}\right),
$$

where the upper sign gives prolate configurations and the lower sign oblate. In the limit $J_{0} \rightarrow \infty, h$ becomes independent of $J_{z}$, and $f$ reduces to the usual distribution function for spherical polytropes. In the limit $J_{0} \rightarrow 0$ for prolate clusters,

\footnotetext{
${ }^{2}$ With the factor of 8 , this quantity is the Newtonian limit of a relativistic measure of the tidal field, the Riemann invariant $R_{\alpha \beta \gamma \delta} R^{\alpha \beta \gamma \delta}$, evaluated in vacuum.
}

$h \rightarrow \delta\left(J_{z}\right)$ (cf. Lake 1981b). This limit is interesting because it produces prolate spindles along the axis.

\section{NUMERICAL METHOD}

The heart of the procedure consists of solving equations (2.11)-(2.13) self-consistently. We begin with an initial guess for $\Phi$ and then integrate equation (2.11) to determine $\rho$ up to the factor $K$ appearing in equation (2.28). Integrating equation (2.9) gives the Legendre coefficients $\rho_{2 l}$. The unknown factor $K$ is then found by requiring that equation (2.13) for the total mass be satisfied. Next we integrate equation (2.12) to determine $\Phi_{2 l}$ from $\rho_{2 l}$. Finally, we reconstruct the updated value of $\Phi$ from equation (2.8). The whole procedure is iterated until convergence. A similar scheme was used by Prendergast \& Tomer (1970) and Wilson (1975) for distribution functions based on distorted Maxwellians.

To carry out the quadratures in $r$ and $x$, we construct a fixed grid in both variables. The radial grid extends from the origin into the vacuum, with successive radial intervals in a fixed ratio. The first zone is chosen to lie well inside the quasihomogeneous core, which is sometimes at quite small radius for very condensed clusters with large values of $n$ or with appreciable prolateness. By adopting the Green's function representation for $\Phi$ via equation (2.6), we can place the outer radius just outside the matter surface if we make the cutoff $L$ large enough. For spherical clusters the surface is at $r=1$. For nonspherical systems the surface typically lies inside $r=1.5$, which we choose as our outer boundary. Given a fixed total number of radial grid points, the grid spacing can now be determined by summing a geometric series.

The angular integrations are all performed by Gaussian quadrature. We do this not only for the high efficiency of Gaussian quadrature for smooth functions. Gaussian quadrature also guarantees the required orthogonality of the terms in Legendre expansions such as equation (2.9). This avoids spurious contributions to the high modes. The choice of Gaussian quadrature dictates that the angular grid points be the $L+1$ positive zeros of $P_{2 L+2}(x)$.

Since we can evaluate the integrands in equations (2.11) and (2.14) at any values of $p_{\perp}$ and $p_{\phi}$, we carry out the quadrature over each variable by Romberg integration. For $1 / 2<n<3 / 2$ the integrands have an integrable singularity and require special treatment (see Appendix A).

Radial quadratures, such as in equations (2.12) and (2.13), are performed by the trapezoidal rule. For high accuracy, it is crucial that the integrands be regularized; for example, $r^{2} d r \rightarrow d r^{3} / 3$.

To evaluate the tidal invariant $I$ in equation (2.27), we calculate the radial derivatives by simple finite differencing and the angular derivatives by using the Legendre expansion (see Appendix B).

For a convergent solution, we require the maximum fractional change in the potential on successive iterations to be less than $10^{-4}$. This typically takes 10 to 20 iterations when we start with crude initial guesses for the potential. A significant number of iterations can be saved during a sequence of calculations in which $J_{0}$ is varied by using the last solution as the initial guess for the next value of $J_{0}$. Once a convergent solution is obtained, we assess its overall accuracy in a number of ways. We require that $\rho_{2 l}(r)$ for $l$ close to $L$ be small compared with $\rho_{0}(r)$, thereby ensuring that we have chosen $L$ large enough. We also check that the virial equation (2.19) is satisfied. Typically it is satisfied to better than $0.1 \%$. 
For nearly spherical clusters, 80 radial zones and 10 angular zones are adequate. (For spherical systems we can set $L=0$ and reproduce the Lane-Emden functions for polytropes to very high accuracy.) For extremely distorted clusters, we use 320 radial zones and 30 angular zones. Such a high resolution run requires about 1 CPU hour on an IBM RS-6000 workstation.

\section{ANALYTIC PRELIMINARIES}

There are a number of straightforward analytic results that help us assess the physical content of the numerical computations.

\subsection{Homogeneous Spheroids}

Homogeneous spheroids provide convenient analytic models for understanding the structure of nonspherical solutions. It is well-known that nonnegative distribution functions of the form $f=f\left(E, J_{z}\right)$ cannot produce homogeneous clusters (see, e.g., Hunter 1975). The required $f$ must depend on at least a third integral of the motion. Such solutions have been independently given by Freeman (1966) and Bisnovatyi-Kogan \& Zel'dovich (1970; see Fridman \& Polyachenko 1984 and Shapiro \& Teukolsky 1987 for an extensive discussion). While the numerical solutions obtained in this paper are for clusters described by just two integrals of the motion, it is still useful to compare their properties with simple homogeneous spheroids.

In this paper, the feature we are most concerned with is the tidal invariant $I$. For a homogeneous cluster with nondimensional density

$$
\rho_{*}=\frac{3}{4 \pi a^{2} c}
$$

we find

$$
I= \begin{cases}192\left(\pi \rho_{*}\right)^{2} A_{1}^{2} & \text { (pole, exterior) } \\ 32\left(\pi \rho_{*}\right)^{2}\left[\left(A_{1}+A_{3}\right)^{2}+A_{1}^{2}+A_{3}^{2}\right] & \text { (equator, exterior) } \\ 32\left(\pi \rho_{*}\right)^{2}\left(2 A_{1}^{2}+A_{3}^{2}\right) & \text { (interior) }\end{cases}
$$

where for prolate clusters

$$
\begin{aligned}
& A_{1}=\frac{1}{e^{2}}-\frac{1-e^{2}}{2 e^{3}} \ln \frac{1+e}{1-e}, \\
& A_{3}=\frac{1-e^{2}}{e^{3}} \ln \frac{1+e}{1-e}-2 \frac{1-e^{2}}{e^{2}},
\end{aligned}
$$

while for oblate clusters

$$
\begin{aligned}
& A_{1}=\frac{\left(1-e^{2}\right)^{1 / 2}}{e^{3}} \sin ^{-1} e-\frac{1-e^{2}}{e^{2}}, \\
& A_{3}=\frac{2}{e^{2}}-\frac{2\left(1-e^{2}\right)^{1 / 2}}{e^{3}} \sin ^{-1} e .
\end{aligned}
$$

Note that for these homogeneous clusters, $I$ is discontinuous at the matter surface.

For a homogeneous sphere, where the nondimensional radius is one, we have

$$
I=\left\{\begin{array}{ll}
\frac{48}{r^{6}} & (\text { exterior, } r>1) \\
24 & \text { (interior, } r<1)
\end{array} .\right.
$$

In the spherical limit $e \rightarrow 0$, the spheroidal expressions (4.2) reduce correctly to equation (4.5).
In the more interesting highly eccentric limit $e \rightarrow 1$, the tidal invariant for a homogeneous prolate spindle has the singular limiting behavior

$$
\begin{aligned}
I_{\text {pole }} & \rightarrow \frac{108}{c^{6}} \frac{1}{\left(1-e^{2}\right)^{2}}, \\
I_{\text {interior }} & \rightarrow I_{\text {eq }} \rightarrow \frac{1}{3} I_{\text {pole }} .
\end{aligned}
$$

The corresponding limit for an oblate pancake is

$$
\begin{aligned}
I_{\mathrm{eq}} & \rightarrow \frac{144}{a^{6}} \frac{1}{\left(1-e^{2}\right)}, \\
I_{\text {interior }} & \rightarrow \frac{1}{2} I_{\mathrm{eq}}, \quad I_{\mathrm{pole}} \rightarrow \frac{3}{4}\left(1-e^{2}\right) I_{\mathrm{eq}} .
\end{aligned}
$$

Note that in the prolate case the peak value of $I$ occurs in the exterior at the pole of the spindle. In the oblate case, the maximum occurs in the equator just outside the matter. More significantly, the singularity is much weaker in the oblate case. In fact, the equations of motion allow all particle trajectories to be integrated right through the singular pancake region. By contrast, particle trajectories are forced to terminate at a prolate spindle singularity.

For spherical systems, the exterior tidal field can never be singular: equation (4.5) is always valid in the exterior independent of the interior density distribution. For nonspherical clusters, however, this need not be true. Homogeneous clusters with $e \rightarrow 1$ provide one example. Below we employ numerical computations to search for further examples.

The occurrence of the maximum value of $I$ in the exterior is a feature of homogeneous clusters. For example, in a spherical polytrope, the central value of $I$ is

$$
I_{\text {center }}=24\left(\frac{\rho_{c}}{\bar{\rho}}\right)^{2},
$$

where $\rho_{c}$ is the central density and $\bar{\rho}$ is the mean density. The exterior values are still given by equation (4.5). Hence, even for small central concentration, the central value exceeds the exterior values. Two key questions we wish to answer for highly prolate, inhomogeneous clusters are therefore: does $I$ blow up as the spindle becomes more pronounced, and if so, does the singularity extend out into the exterior, or is it confined to the core? To address these questions, we have to rely on numerical simulations.

\subsection{Inhomogeneous Spindles}

In general, whenever $h\left(J_{z}\right) \rightarrow \delta\left(J_{z}\right)$, a prolate spindle singularity is generated along the axis. An easy way to see this is to work in cylindrical coordinates $(R, z)$ and to recast equation (2.5) in the form of an integral over $E$ and $J_{z}$ :

$$
\rho(R, z)=\frac{2 \pi}{R} m \int_{\Phi}^{E_{\max }} d E \int_{J_{z^{2}<2(-\Phi+E) R^{2}}} d J_{z} f\left(E, J_{z}\right)
$$

(see, e.g., Binney \& Tremaine 1987). Employing equation (1.1) with $h$ equal to a $\delta$-function, we find

$$
\rho(R, z)=\frac{2 \pi m}{R} \int_{\Phi}^{E_{\max }} d E g(E) .
$$

Thus if $g(E)$ is a smooth function, the integral in equation (4.10) is regular, and the density spikes up on the axis. Note however that the total mass in the singularity is finite because $\rho \propto 1 / R$. By Poisson's equation, the second derivatives of $\Phi$ blow up on 
TABLE 1

Properties of Prolate Sequences ${ }^{a}$

\begin{tabular}{|c|c|c|c|c|c|c|c|}
\hline$n$ & $J_{0}$ & $e$ & $-E_{\text {tot }}$ & $T_{\text {rot }} /|W|^{\mathbf{b}}$ & $f_{<}$ & $\rho_{c}^{c}$ & $I_{\max }^{d}$ \\
\hline $0.6 \ldots \ldots \ldots \ldots$ & $\begin{array}{l}1 \\
0.1 \\
0.01\end{array}$ & $\begin{array}{l}0 \\
0.258 \\
0.820 \\
0.887\end{array}$ & $\begin{array}{l}15 / 44 \\
0.341 \\
0.344 \\
0.345\end{array}$ & $\begin{array}{c}1 / 6 \\
0.161 \\
0.0575 \\
0.0049\end{array}$ & $\begin{array}{l}0.69 \\
0.64 \\
0.095 \\
0.004\end{array}$ & $\begin{array}{c}0.495 \\
0.523 \\
1.67 \\
15.0\end{array}$ & $\begin{array}{l}1.04(+2) \\
1.16(+2) \\
1.61(+3) \\
1.40(+5)\end{array}$ \\
\hline $1.5 \ldots \ldots \ldots \ldots$ & $\begin{array}{l}1^{\infty} \\
0.1 \\
0.01\end{array}$ & $\begin{array}{l}0 \\
0.231 \\
0.775 \\
0.861\end{array}$ & $\begin{array}{c}3 / 7 \\
0.431 \\
0.469 \\
0.497\end{array}$ & $\begin{array}{l}1 / 6 \\
0.162 \\
0.065 \\
0.0077\end{array}$ & $\begin{array}{l}0.44 \\
0.39 \\
0.092 \\
0.008\end{array}$ & $\begin{array}{r}1.43 \\
1.53 \\
5.36 \\
55.1\end{array}$ & $\begin{array}{l}8.76(+2) \\
9.85(+2) \\
1.53(+4) \\
1.89(+6)\end{array}$ \\
\hline $2.5 \ldots \ldots \ldots \ldots$ & \begin{tabular}{l}
\multicolumn{1}{c}{${ }^{\infty}$} \\
0.1 \\
0.01 \\
0.0001
\end{tabular} & $\begin{array}{l}0 \\
0.199 \\
0.719 \\
0.815 \\
0.815\end{array}$ & $\begin{array}{c}3 / 5 \\
0.606 \\
0.762 \\
0.951 \\
0.997\end{array}$ & \begin{tabular}{l}
\multicolumn{1}{c}{$1 / 6$} \\
0.162 \\
0.078 \\
0.013 \\
0.00053
\end{tabular} & $\begin{array}{l}0.24 \\
0.23 \\
0.099 \\
0.014 \\
0.0007\end{array}$ & $\begin{array}{c}5.57 \\
5.93 \\
27.4 \\
438 . \\
5.47(3)\end{array}$ & $\begin{array}{l}1.34(+4) \\
1.51(+4) \\
3.72(+5) \\
1.17(+8) \\
1.88(+10)\end{array}$ \\
\hline $3.5 \ldots \ldots \ldots \ldots$ & $\begin{array}{l}1^{\infty} \\
0.1 \\
0.01\end{array}$ & $\begin{array}{l}0 \\
0.160 \\
0.644 \\
0.739\end{array}$ & $\begin{array}{l}1 \\
1.01 \\
1.64 \\
5.19\end{array}$ & $\begin{array}{r}1 / 6 \\
0.164 \\
0.100 \\
0.032\end{array}$ & $\begin{array}{l}0.19 \\
0.18 \\
0.11 \\
0.034\end{array}$ & $\begin{array}{l}36.4 \\
38.6 \\
298 . \\
5.65(+4)\end{array}$ & $\begin{array}{l}5.70(+5) \\
6.43(+5) \\
4.03(+7) \\
1.78(+12)\end{array}$ \\
\hline
\end{tabular}

a The distribution function $f\left(E, J_{z}\right)$ is defined by eqs. (1.1), (2.28), and (2.29). The quantity $J_{0}$ is in units of $G M /\left|E_{\max }\right|^{1 / 2}, E_{\text {tot }}$ is in units of $M\left|E_{\max }\right|, \rho_{c}$ is in units of $M\left(G M /\left|E_{\max }\right|\right)^{-3}$, and $I_{\max }$ is in units of $\left|E_{\max }\right|^{6} /(G M)^{4}$

This is the maximal value; in general it should be multiplied by $(2 \chi-1)^{2}$.

c The central density $\rho_{c}$ is also the maximum density for the clusters listed here.

d The quantity $I_{\max }$ is the maximum value of the tidal invariant and occurs at the center of all clusters listed here.

the axis in the same way as $\rho$. We thus expect that $I$ should be singular in the region of the matter spindle. Does the singularity in $I$ extend further out? Our numerical calculations will address this question below.

A spindle singularity may be present in a cluster even when the global eccentricity defined by equation (2.23) has only a moderate value. For example, set $g(E)=\delta\left(E-E_{\max }\right)$ in equa- tion (4.10), which would have given an $n=\frac{1}{2}$ spherical polytrope for $h$ constant. Then $\rho=1 / R$ everywhere, up to a constant factor. The corresponding Legendre coefficients are

$$
\rho_{0}(r)=\frac{\pi}{2 r}, \quad \rho_{2}(r)=\frac{5 \pi}{8 r},
$$

so by equations (2.22) and (2.23) the eccentricity is exactly $1 / 2$.

TABLE 2

Properties of Oblate Sequences ${ }^{\mathrm{a}}$

\begin{tabular}{|c|c|c|c|c|c|c|c|}
\hline$n$ & $J_{0}$ & $e$ & $-E_{\mathrm{tot}}$ & $T_{\text {rot }} /|W|^{b}$ & $f_{<}$ & $\rho_{c}{ }^{c}$ & $I_{\max }{ }^{\mathrm{d}}$ \\
\hline $0.6 \ldots \ldots \ldots \ldots$ & $\begin{array}{l}\infty \\
1 \\
0.5 \\
0.4\end{array}$ & $\begin{array}{l}0 \\
0.269 \\
0.577 \\
0.758\end{array}$ & $\begin{array}{l}15 / 44 \\
0.341 \\
0.340 \\
0.339\end{array}$ & $\begin{array}{l}1 / 6 \\
0.175 \\
0.205 \\
0.243\end{array}$ & $\begin{array}{l}0.69 \\
0.74 \\
0.73 \\
0.52\end{array}$ & $\begin{array}{l}0.495 \\
0.465 \\
0.361^{*} \\
0.259^{*}\end{array}$ & $\begin{array}{l}1.04(+2) \\
9.25(+1) \\
9.99(+1)^{*} \\
2.08(+2)^{*}\end{array}$ \\
\hline $1.5 \ldots \ldots \ldots \ldots$ & $\begin{array}{l}\infty \\
1 \\
0.5 \\
0.4\end{array}$ & $\begin{array}{l}0 \\
0.248 \\
0.523 \\
0.688\end{array}$ & $\begin{array}{c}3 / 7 \\
0.426 \\
0.417 \\
0.407\end{array}$ & $\begin{array}{c}1 / 6 \\
0.174 \\
0.199 \\
0.229\end{array}$ & $\begin{array}{l}0.44 \\
0.40 \\
0.55 \\
0.61\end{array}$ & $\begin{array}{l}1.43 \\
1.34 \\
1.03 \\
0.757 *\end{array}$ & $\begin{array}{l}8.76(+2) \\
7.70(+2) \\
4.92(+2) \\
4.05(+2)^{*}\end{array}$ \\
\hline $2.5 \ldots \ldots \ldots \ldots$ & $\begin{array}{l}1^{\infty} \\
0.5 \\
0.4 \\
0.33\end{array}$ & $\begin{array}{l}0 \\
0.210 \\
0.447 \\
0.588 \\
0.781\end{array}$ & $\begin{array}{c}3 / 5 \\
0.592 \\
0.567 \\
0.542 \\
0.492\end{array}$ & $\begin{array}{l}1 / 6 \\
0.171 \\
0.190 \\
0.211 \\
0.257\end{array}$ & $\begin{array}{l}0.24 \\
0.25 \\
0.31 \\
0.38 \\
0.58\end{array}$ & $\begin{array}{l}5.57 \\
5.19 \\
3.99 \\
3.01 \\
1.53^{*}\end{array}$ & $\begin{array}{l}1.34(+4) \\
1.16(+4) \\
7.04(+3) \\
4.44(+3) \\
1.71(+3)^{*}\end{array}$ \\
\hline $3.5 \ldots$ & $\begin{array}{l}\infty \\
1 \\
0.5 \\
0.4 \\
0.3\end{array}$ & $\begin{array}{l}0 \\
0.168 \\
0.356 \\
0.468 \\
0.726\end{array}$ & $\begin{array}{l}1 \\
0.981 \\
0.920 \\
0.864 \\
0.688\end{array}$ & $\begin{array}{c}1 / 6 \\
0.170 \\
0.181 \\
0.193 \\
0.242\end{array}$ & $\begin{array}{l}0.19 \\
0.19 \\
0.20 \\
0.21 \\
0.34\end{array}$ & $\begin{array}{c}36.4 \\
33.8 \\
25.8 \\
19.9 \\
7.07\end{array}$ & $\begin{array}{l}5.70(+5) \\
4.91(+5) \\
2.88(+5) \\
1.72(+5) \\
2.45(+4)\end{array}$ \\
\hline
\end{tabular}

a The distribution function $f\left(E, J_{z}\right)$ is defined by equations (1.1), (2.28), and (2.29). The quantity $J_{0}$ is in units of $G M /\left|E_{\max }\right|^{1 / 2}, E_{\text {tot }}$ is in units of $M\left|E_{\max }\right|, \rho_{c}$ is in units of $M\left(G M /\left|E_{\max }\right|\right)^{-3}$, and $I_{\max }$ is in units of $\left|E_{\max }\right|^{6} /(G M)^{4}$.

${ }^{b}$ This is the maximal value; in general it should be multiplied by $(2 \chi-1)^{2}$.

c The central density $\rho_{c}$ is also the maximum density for all the clusters except where indicated by an asterisk, when it occurs on an equatorial ring.

${ }^{d}$ The quantity $I_{\max }$ is the maximum value of the tidal invariant, and occurs at the center of all the clusters except where indicated by an asterisk, when it occurs on an equatorial ring. 
We will see more realistic examples of this behavior in the numerical cases below.

\section{NUMERICAL RESULTS}

We have constructed numerical models for the distribution function defined in equations (2.28) and (2.29). We have surveyed a range of polytropic indicies including $n=0.6,1.5,2.5$, and 3.5. Results are summarized in Tables 1 and 2. The case of $n=0.6$ is the closest to a homogeneous cluster, while the case of $n=3.5$ is the most centrally condensed.

In Figure 1 we plot density contours for selected prolate clusters with $n=0.6$ along a sequence of decreasing $J_{0}$. As $J_{0}$ decreases and the depletion of angular momentum increases, the density spikes up along the axis. The corresponding plot of particle positions projected into a plane through the axis is given in Figure 2. We see little evidence in the particle plot for a spindle singularity, and indeed the mean eccentricity of even the lowest $J_{0}$ clusters is less than 0.9 . However the tidal invariant $I$ is growing rapidly along the sequence (Table 1). The fraction of particles (i.e., mass) inside the singular region decreases as the spindle becomes more pronounced. Move out along the axis from the center until the density has fallen to half its central value. Define $f_{<}$to be the fraction of particles inside a sphere out to this radius. As shown in Table $1, f_{<}$ decreases sharply along the sequence, in agreement with the analytic discussion in $\S 4.2$.

Figure 3 shows density contours for a sequence of oblate clusters with $n=0.6$. As $J_{0}$ decreases and the angular momentum distribution for $J_{z}$ is now enhanced, the configuration becomes increasingly flattened. Eventually the location of the maximum density moves out from the origin, and a condensed equatorial ring appears. Once again, as shown in Figure 4, projected particle plots do not reveal much of this structure. For $J_{0} \lesssim 0.3$, the density along the axis falls to zero, and the configuration becomes toroidal. We have not studied these configurations in detail.

Density contours for the more centrally condensed case of $n=2.5$ are shown in Figure 5 for prolate clusters. As the spindle emerges on the axis with decreasing $J_{0}$, the low-density outer regions are hardly affected. The accumulation of stars on the axis is now evident in the particle plot in Figure $6 d$. However, once again the mean eccentricity is not very extreme, $e \simeq 0.8$. By contrast with the mean eccentricity, the tidal invariant grows dramatically as the core becomes increasingly prolate. In Figure 7 we plot the tidal invariant in a quadrant of the $x-z$ plane for an extreme $J_{0}=0.01$ configuration. The plot clearly shows the dramatic spike in $I$ along the axis. The maximum occurs at the center in this centrally condensed example.
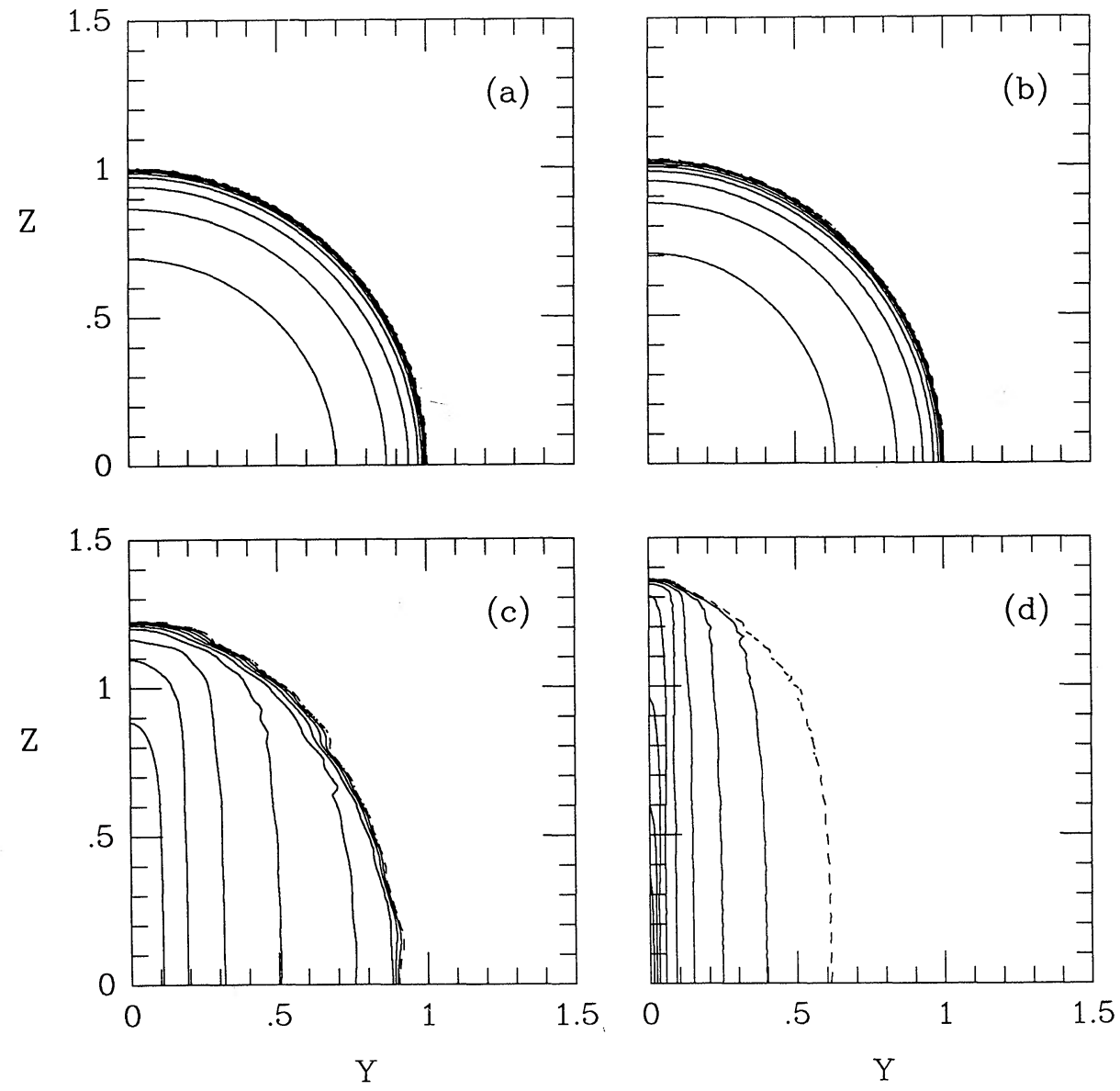

FIG. 1.-Density contours in a meridional plane for a sequence of prolate clusters with $n=0.6$. The angular momentum parameter $J_{0}$ is in units of $G M /\left|E_{\max }\right|^{1 / 2}$ and has the values $\infty(a), 1(b), 0.1(c)$, and $0.01(d)$. The density falls by a factor of 1.7 on successive contour lines. The surface is shown by a dashed line. The coordinates are in units of $G M /\left|E_{\max }\right|$. The density profile for this value of $n$ is fairly homogeneous. 

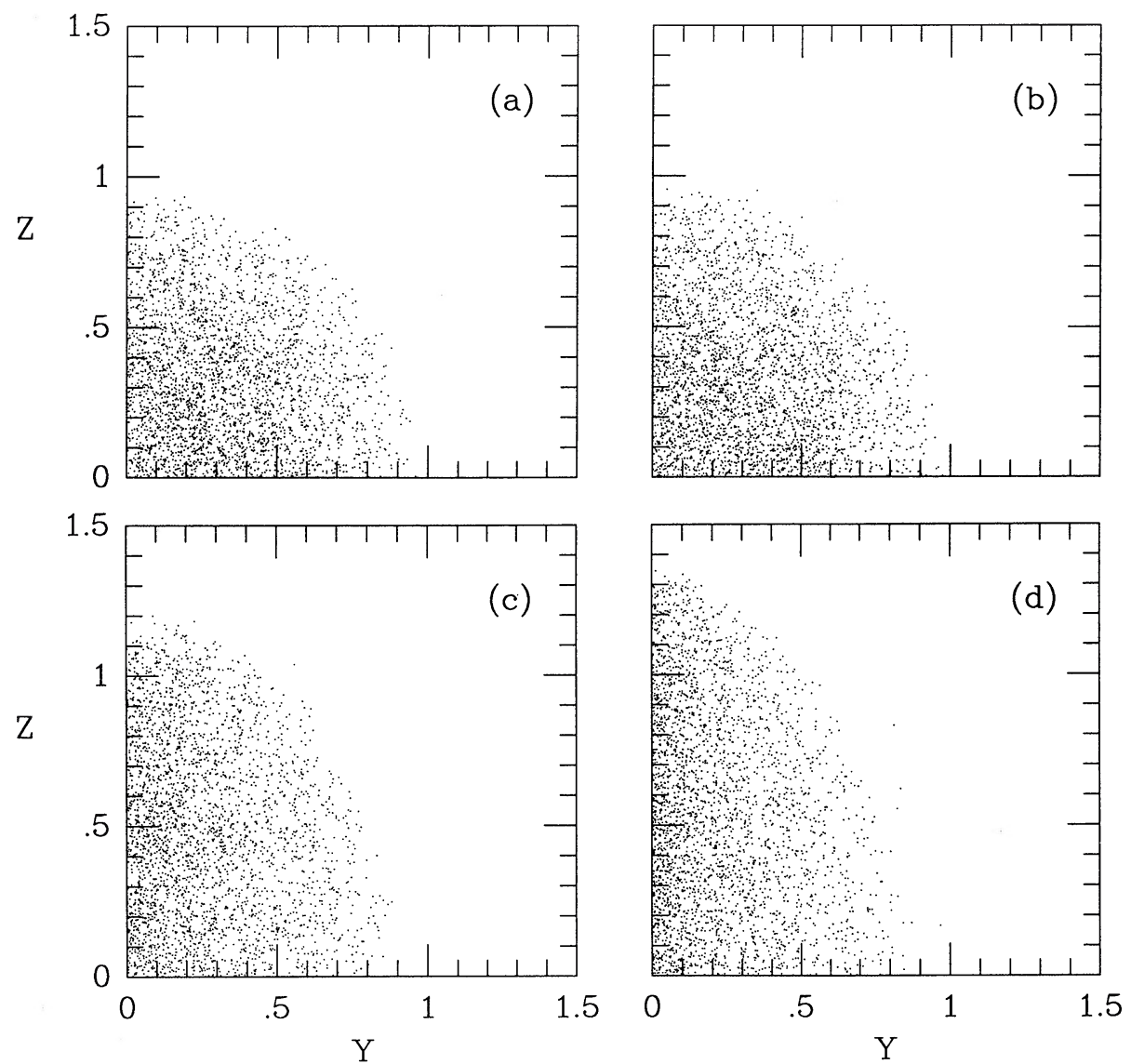

Fig. 2.-Snapshots of the particle positions projected onto a meridional plane for the prolate sequence shown in Fig. 1. The growth of the spindle along the sequence, which is evident in Fig. 1, is less discernible in these particle plots.
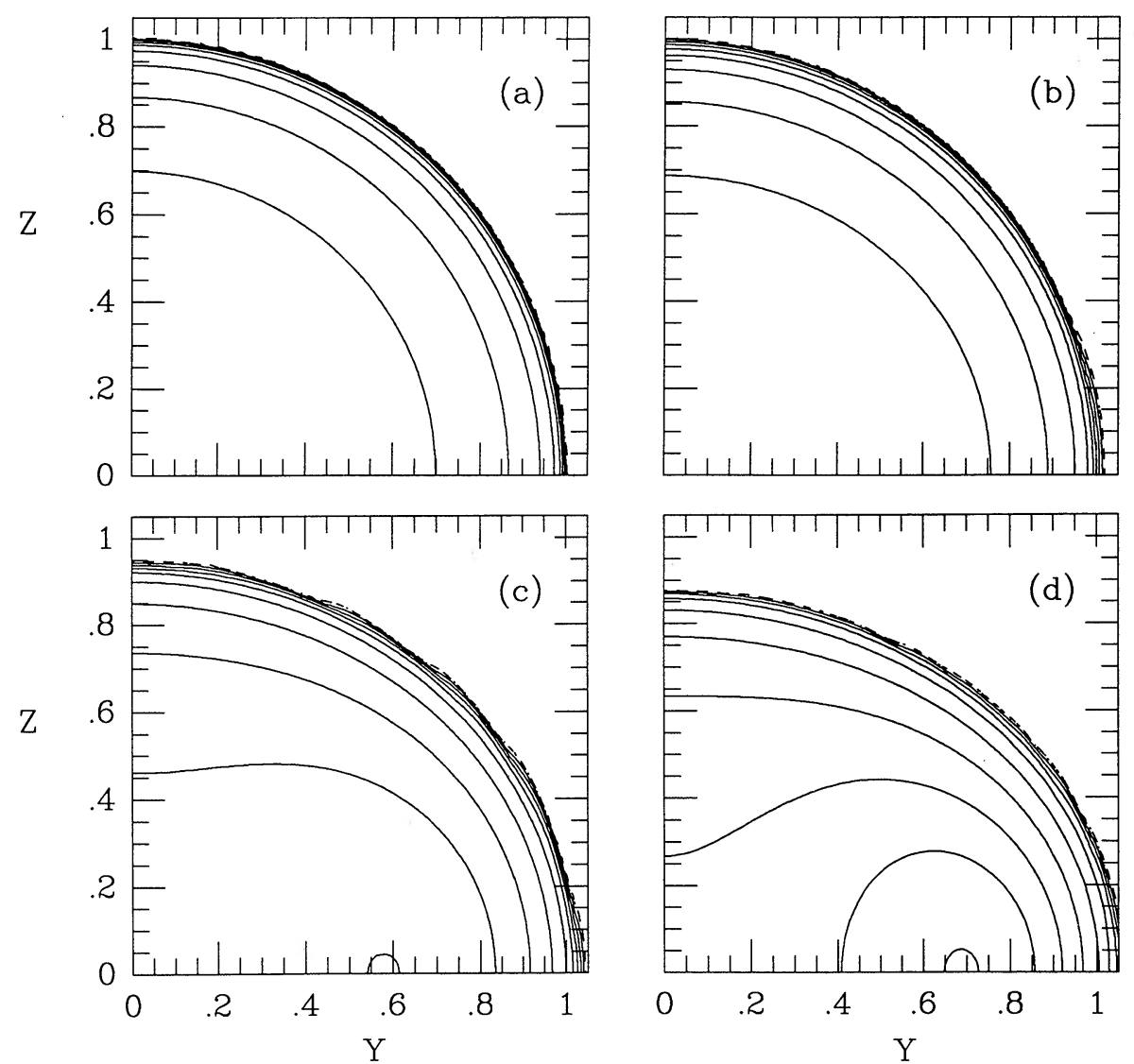

FIG. 3--Density contours in a meridional plane for a sequence of oblate clusters with $n=0.6$. The angular momentum parameter $J_{0}$ has the values $\infty(a), 1(b)$ $0.5(c)$, and $0.4(d)$. The labeling is as in Fig. 1. The density profile for this value of $n$ is fairly homogeneous. Note the appearance of an equatorial ring in cases $(c)$ and $(d)$. 

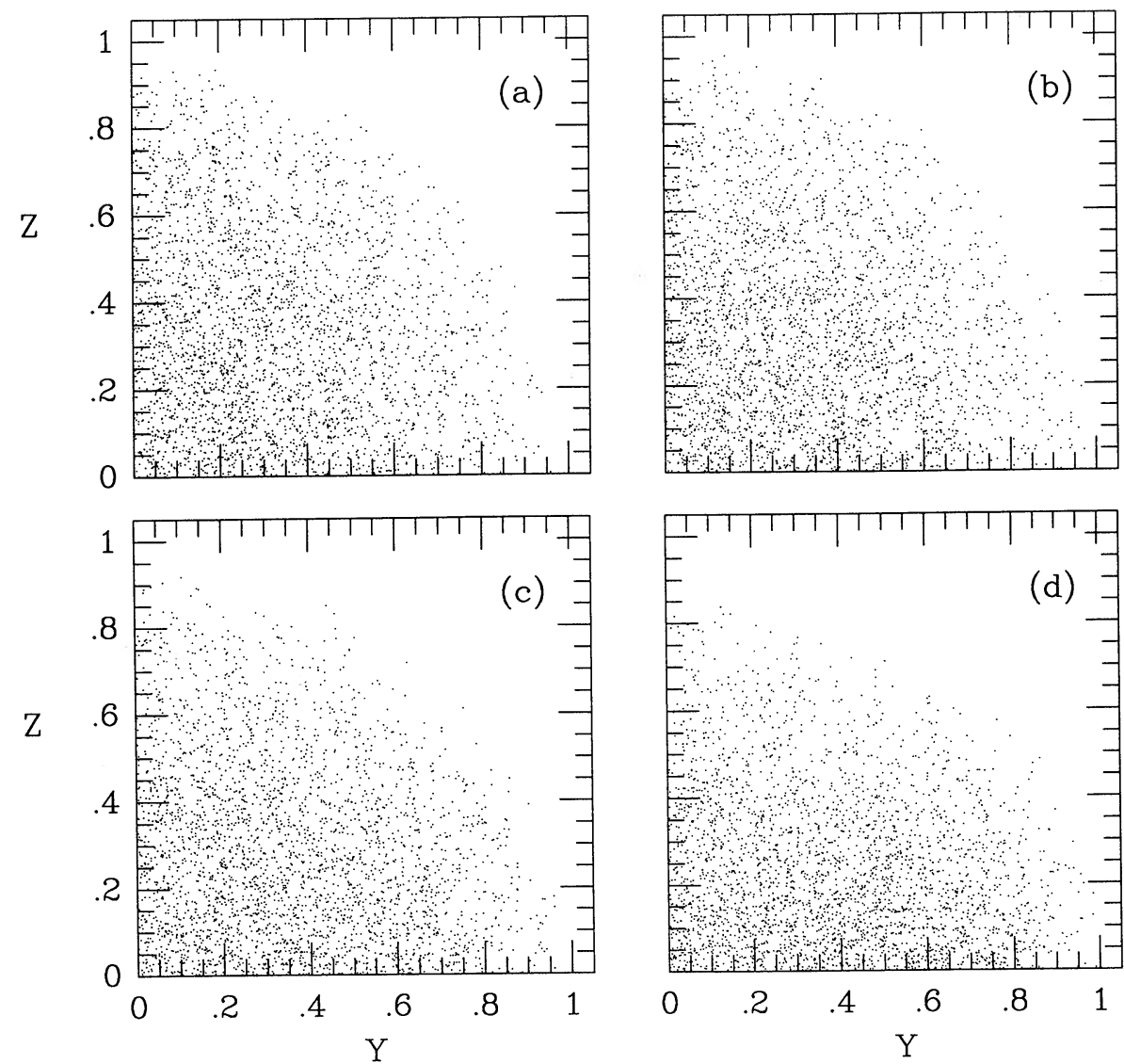

FIG. 4.-Snapshots of the particle positions projected onto a meridional plane for the oblate sequence shown in Fig. 3. The growth of the equatorial ring along the sequence, which is evident in Fig. 3, is less discernible in these particle plots.
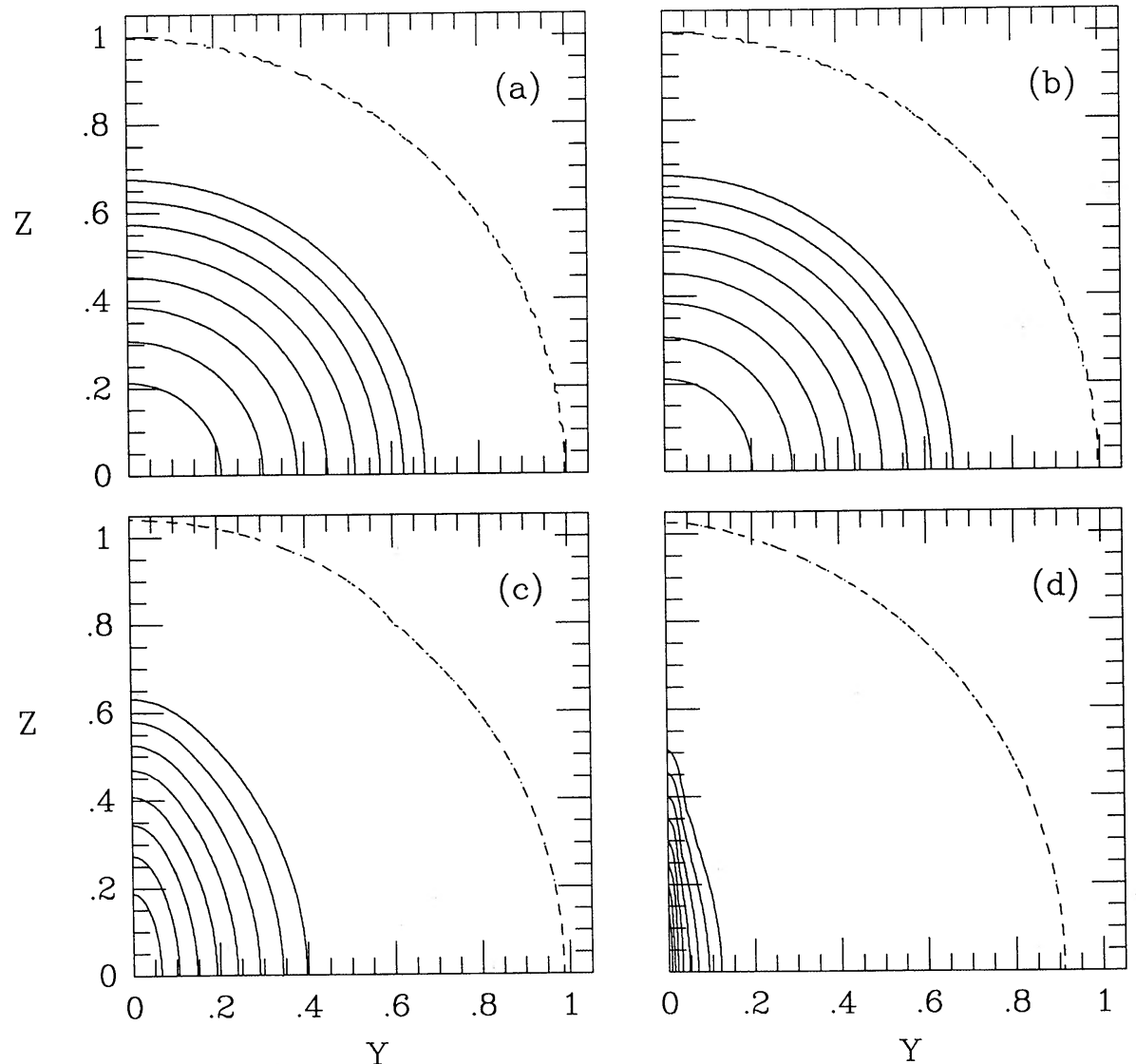

FIG. 5.-Density contours in a meridional plane for a sequence of prolate clusters with $n=2.5$. The values of the angular momentum parameter and the labeling are the same as in Fig. 1 . The density profile for this value of $n$ is quite centrally condensed. The spindle that forms in the extreme cases is confined to the central region. 

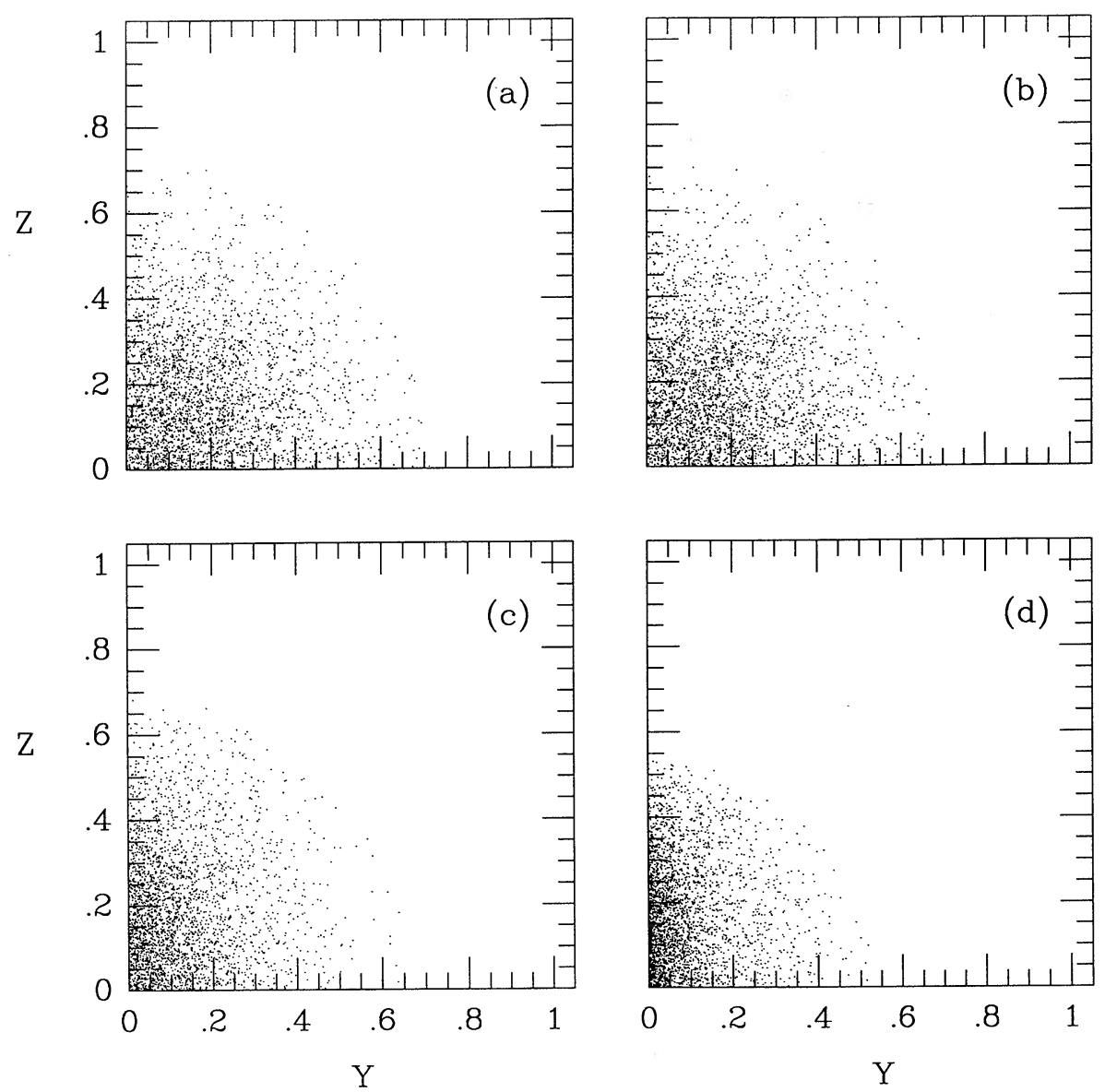

Fig. 6.-Snapshots of the particle positions projected onto a meridional plane for the prolate sequence shown in Fig. 5. The growth of the spindle along the sequence, which is evident in Fig. 5, is less pronounced in these particle plots.

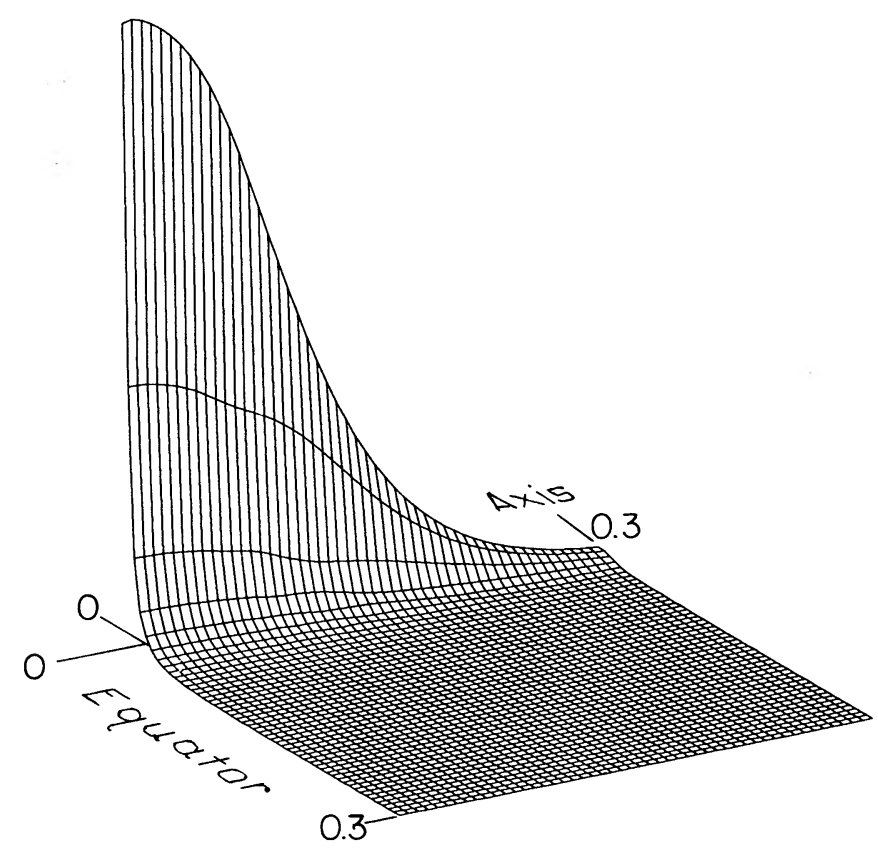

Fig. 7.-Profile of the tidal invariant $I$ in a quadrant of the $x-z$ plane for $n=2.5$ and $J_{0}=0.01$, the extreme prolate case shown in Figs. $1 d$ and $2 d$. The coordinates are in units of $G M /\left|E_{\max }\right|$. The peak value of $I$ is $1.17 \times 10^{8}$ in units of $\left|E_{\max }\right|^{6} /(G M)^{4}$. The extremely steep tidal field along the axis foreshadows the ultimate appearance of a singularity as $J_{0} \rightarrow 0$ along the sequence.
In Figure 8 we compare the tidal invariant profiles along the axis for the prolate $n=0.6$ and $n=2.5$ cases. The more homogeneous $n=0.6$ models are reminiscent of the homogenous spheroids discussed in $\S 4.1$ : the interior values of $I$ are roughly constant; they are comparable in magnitude to the exterior surface values; and there is an abrupt change in the value at the surface. It is most significant that as $J_{0} \rightarrow 0$ and $h \rightarrow \delta\left(J_{z}\right), I$ blows up to infinity along the axis. The singularity in $I$ is not confined to the interior, but clearly extends to the region just outside the surface. This feature is most easily discernible in the plot for $n=0.6$, but is also true for $n=2.5$. Such vacuum singularities do not arise for spherical systems, where the exterior value of $I$ is always given by equation (4.5).

Figure 9 shows density contours for the oblate sequence with $n=2.5$. Again the flattening with decreasing $J_{0}$ is most pronounced in the central regions. As with the $n=0.6$ sequence, extreme cases show the formation of equatorial rings. These rings appear before the mean eccentricity is appreciable (Table 2). Particle plots for these oblate configurations are shown in Figure 10.

The tidal invariant for the $J_{0}=0.5$ oblate cluster with $n=2.5$ is plotted in Figure 11. The profile rises abruptly in the equatorial plane, but is not nearly as extreme as the prolate case. This typifies the behavior of oblate systems.

Insight into the effect of varying the parameter $J_{0}$ to deplete or enhance the $J_{z}$-distribution is provided in Figure 12. There we plot the profile of $2 v_{\phi}^{2} / v_{\perp}^{2}$ along a radius in the equatorial plane. This ratio is identically equal to one for an isotropic 


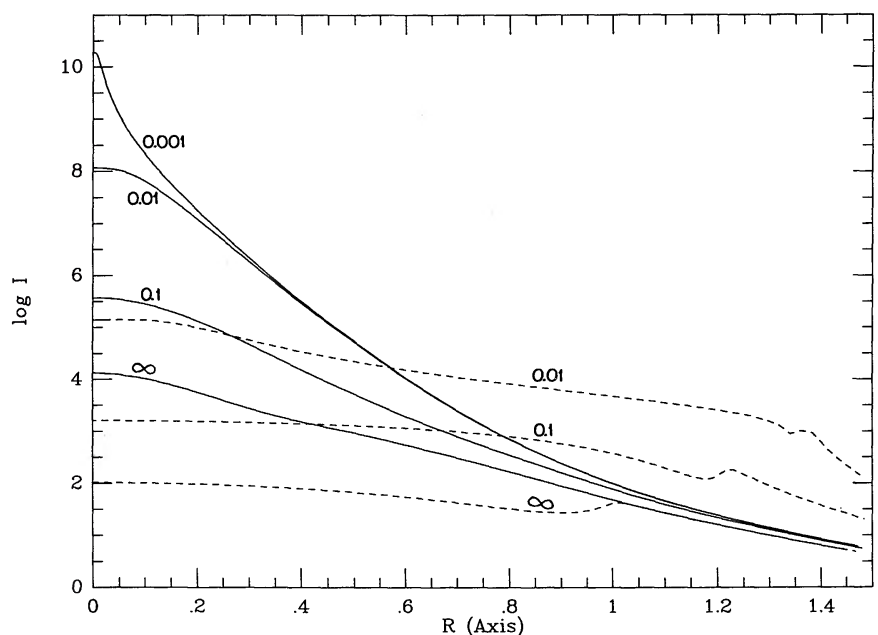

Fig. 8.-Comparison of the tidal invariant profiles along the axis for two prolate sequences. The solid lines show the $n=2.5$ sequence, while the dashed lines show the $n=0.6$ sequence. Each curve is labeled by the value of $J_{0}$, in units of $G M /\left|E_{\max }\right|^{1 / 2}$. The quantity $I$ is in units of $\left|E_{\max }\right|^{6} /(G M)^{4}$, while the radial coordinate $R$ is in units of $G M /\left|E_{\max }\right|$.

distribution function, $h=$ constant. For prolate clusters, where angular momentum is depleted, we see that the ratio dips below unity, while for oblate clusters the ratio rises above unity. At the origin and everywhere along the axis, the ratio is always unity when $f=f\left(E, J_{z}\right)$. The ratio also returns to unity at the surface for smooth distribution functions of this form. However the rise to unity is increasingly abrupt at the surface as $J_{0}$ decreases and vanishes altogether when $J_{0}=0$. In making the plot for the most extreme prolate clusters, the ratio does not return all the way to one at the surface because of insufficient radial grid resolution there.

\section{CONCLUSION}

We have constructed sequences of nonspherical equilibrium clusters that tend toward prolate singular states. The formation of spindle singularities in the Newtonian examples considered here is due to the depletion of angular momentum near the symmetry axis. This depletion allows stars to accumulate there, although the total mass involved is small. While the resulting density enhancement is confined to the region near the axis, the influence of the spindle is felt much further out through its tidal gravitational field. It is interesting that centrally condensed prolate clusters may contain strong-field regions even though their mean eccentricity is not extreme.

The formation and properties of singularities is an important topical issue in general relativity. The cosmic censorship hypothesis, which underpins the use of general relativity to describe gravitational collapse, is still unproven after more than 20 years. Equilibrium solutions for relativistic star clusters can provide a testing ground for exploring this issue. The
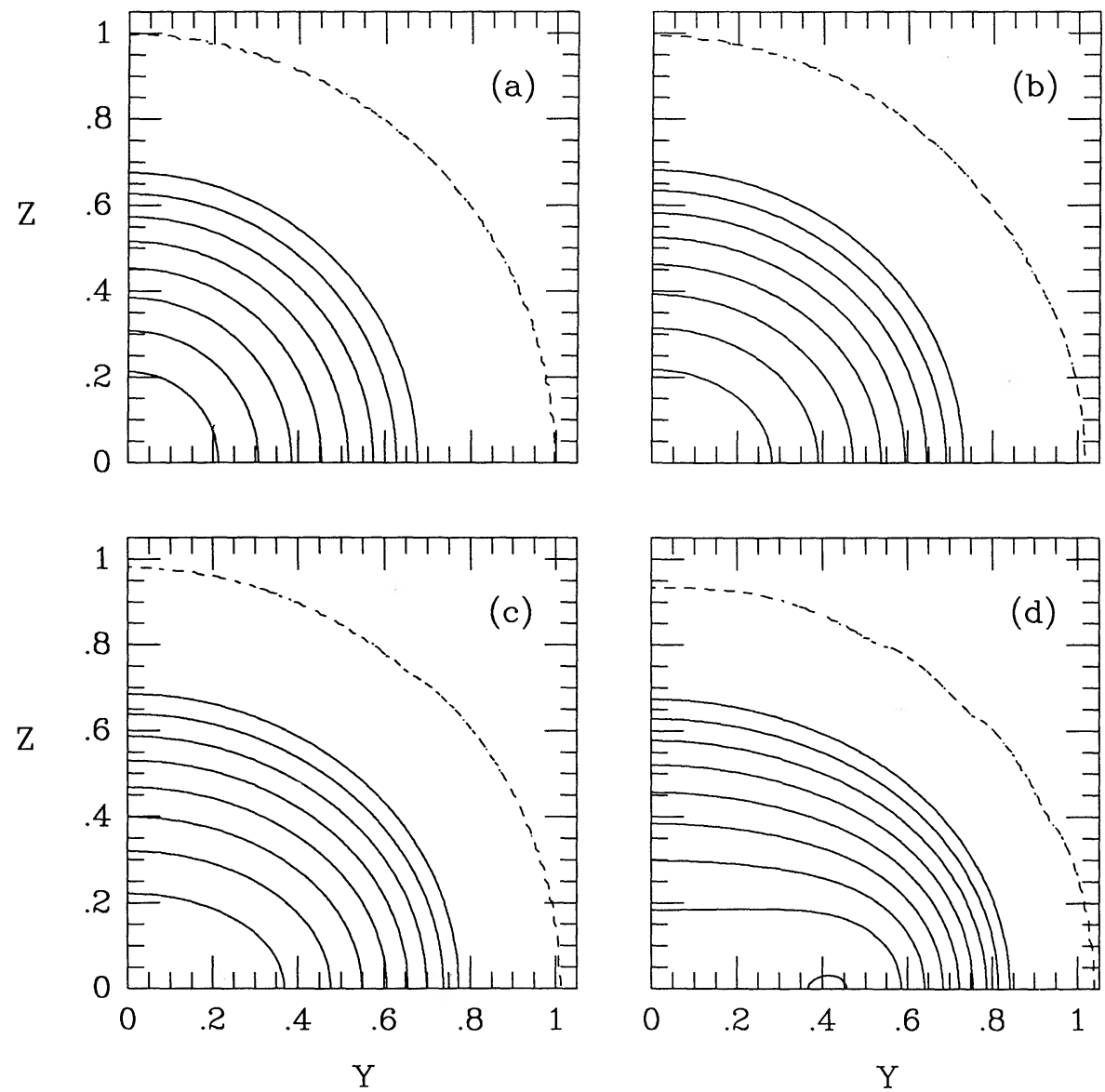

Fig. 9.-Density contours in a meridional plane for a sequence of oblate clusters with $n=2.5$. The angular momentum parameter $J_{0}$ has the values $\infty(a), 0.5(b)$ $0.4(c)$, and $0.33(d)$. The labeling is as in Fig. 1. This sequence is quite centrally condensed. Note the appearance of an equatorial ring in $(d)$. 

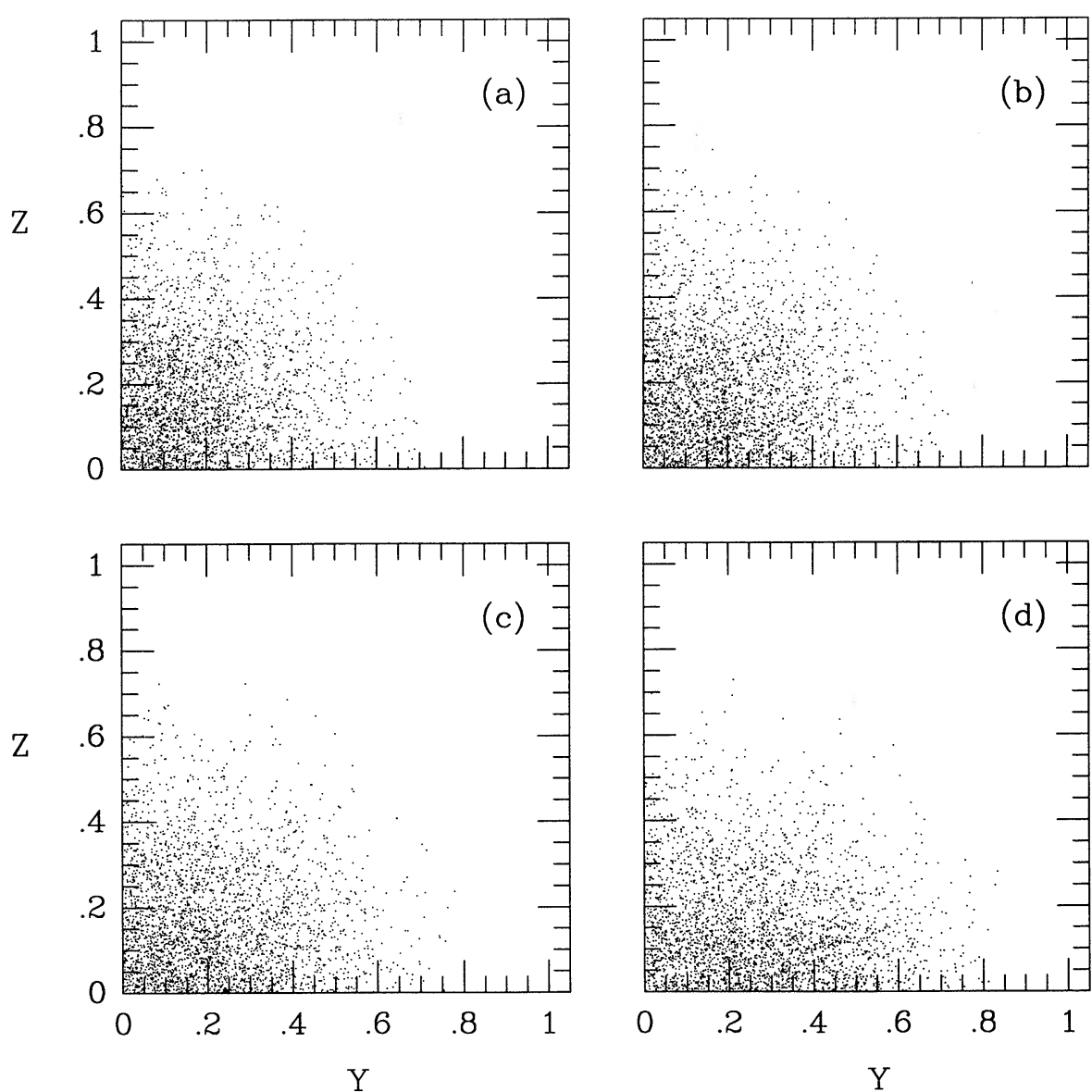

Fig. 10. - Snapshots of the particle positions projected onto a meridional plane for the oblate sequence shown in Fig. 9.

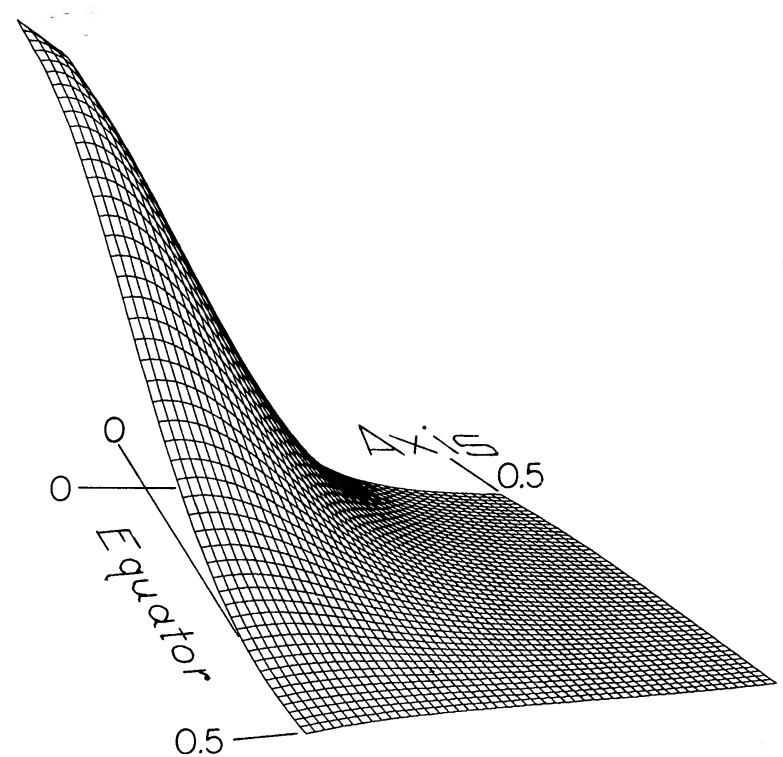

Fig. 11.-Profile of the tidal invariant $I$ in a quadrant of the $x-z$ plane for $n=2.5$ and $J_{0}=0.5$, the oblate case shown in Figs. $9 b$ and $10 b$. Labeling is the same as in Fig. 8. The peak value of $I$ is $7.04 \times 10^{3}$. The tidal invariant is steep along the equatorial plane.

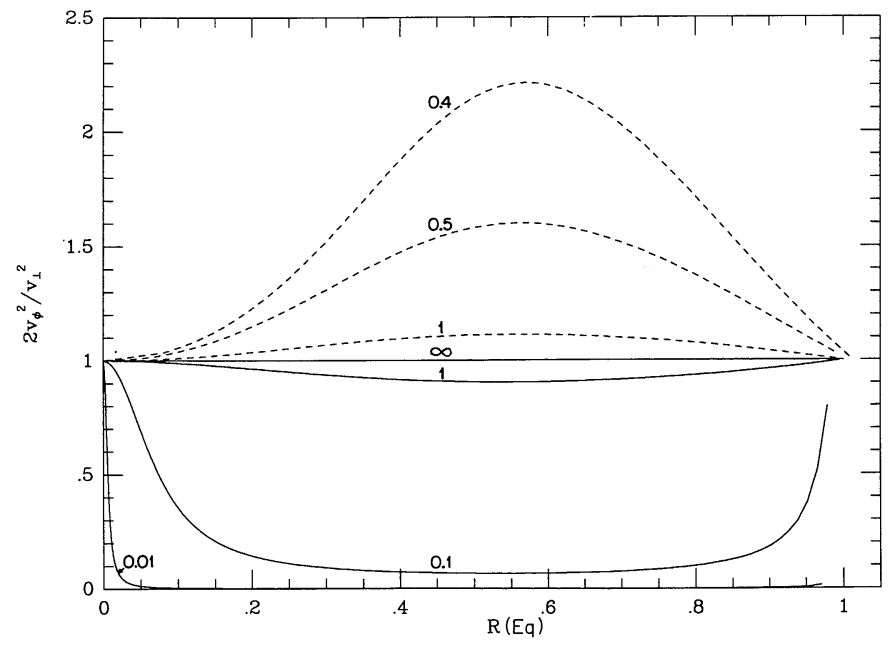

FIG. 12.-Profile of the velocity dispersion ratio along an equatorial radius for $n=2.5$ sequences. The solid lines show prolate clusters, the dashed lines oblate clusters. Curves are labeled by the value of the angular momentum parameter $J_{0}$, in units of $G M /\left|E_{\max }\right|^{1 / 2}$. The radial coordinate $R$ is in units of $G M /\left|E_{\max }\right| \cdot$ 
methods used in this paper for constructing equilibrium clusters can be generalized straightforwardly for relativistic systems, and we plan to do so.
This work has been supported in part by National Science Foundation grants AST 90-15451 and PHY 90-07834, and NASA grant NAGW-2364 at Cornell University.

\section{APPENDIX A}

\section{REGULARIZING EQUATION (2.11) FOR $1 / 2<n<3 / 2$}

To deal with the integrable singularity in equation (2.11) when $1 / 2<n<3 / 2$, we make a change of variable in the integrands. (A similar transformation is used in equation 2.14.) To motivate this change, rewrite equation (2.11) in the form

$$
\rho=\int_{0}^{p_{\perp}^{\max }} d p_{\perp} p_{\perp} f_{\perp}\left(p_{\perp}\right)
$$

where

$$
f_{\perp}\left(p_{\perp}\right)=\int_{0}^{p_{\phi}^{\max }} d p_{\phi} f_{\phi}\left(p_{\phi}\right)
$$

and where

$$
\begin{aligned}
f_{\phi}\left(p_{\phi}\right) & =g(E) h\left(J_{z}\right) \\
& =K\left[-\left(1+\Phi+\frac{1}{2} p_{\perp}^{2}+\frac{1}{2} p_{\phi}^{2}\right)\right]^{n-3 / 2} h\left(J_{z}\right) \\
& =K\left[\frac{1}{2}\left(p_{\phi}^{\max }\right)^{2}-\frac{1}{2} p_{\phi}^{2}\right]^{n-3 / 2} h\left(J_{z}\right) .
\end{aligned}
$$

It is clear that the integrand in equation (A3) blows up at the upper limit of integration for $n<3 / 2$. We can regularize the integrand by changing the variable of integration via

$$
t=\left(1-\frac{p_{\phi}}{p_{\phi}^{\max }}\right)^{n-1 / 2}
$$

This gives

$$
f_{\perp}\left(p_{\perp}\right)=\left(\frac{1}{2}\right)^{n-3 / 2} \frac{1}{n-1 / 2}\left(p_{\phi}^{\max }\right)^{2 n-2} \int_{0}^{1} d t\left(2-t^{1 /(n-1 / 2)}\right)^{n-3 / 2} h\left(J_{z}\right),
$$

where the integral in equation (A5) is now regular. However, since

$$
\left(p_{\phi}^{\max }\right)^{2 n-2}=\left[\left(p_{\perp}^{\max }\right)^{2}-p_{\perp}^{2}\right]^{n-1},
$$

$p_{\perp}$ still blows up at the upper limit of integration in equation (A1) when $n<1$. We can regularize this singularity by the change of variable

$$
s=\left(1-\frac{p_{\perp}}{p_{\perp}^{\max }}\right)^{n} .
$$

In practice, rather than carrying out the substitutions (A4) and (A7) analytically, we implement them numerically in the Romberg quadrature routine (see Press et al. $1986 \S 4.4$ ). Even with these substitutions, the quadrature for $1 / 2<n<3 / 2$ is substantially slower than for $5>n \geq 3 / 2$.

\section{APPENDIX B}

\section{EVALUATION OF I IN EQUATION (2.27)}

From the Legendre expansion (2.8) for $\Phi$, we can get the Legendre expansion of $\Phi_{, x}$ :

$$
\Phi_{, x}=\sum_{l=0}^{\infty} \Psi_{2 l}(r) P_{2 l}(x),
$$

where the expansion coefficients $\Psi_{2 l}$ can be obtained from the downward recurrence relation

$$
\begin{aligned}
& \Psi_{2 L+1}=0 \\
& \Psi_{2 l-1}=(4 l-1)\left(\frac{\Psi_{2 l+1}}{4 l+3}+\Phi_{2 l}\right), \quad l=L, \ldots, 1 .
\end{aligned}
$$


This recurrence relation follows from the relation

$$
(2 l+1) P_{l}(x)=P_{l+1}^{\prime}(x)-P_{l-1}^{\prime}(x)
$$

for Legendre polynomials.

Using Legendre's equation, the term involving second derivatives in $x$ in equation (2.27) can be rewritten as follows:

$$
\begin{aligned}
\left(1-x^{2}\right) \Phi_{, x x} & =\sum_{l=0}^{\infty} \Phi_{2 l}(r)\left[2 x P_{2 l}(x)-2 l(2 l+1) P_{2 l}(x)\right] \\
& =2 x \Phi_{, x}-\sum_{l=0}^{\infty} 2 l(2 l+1) \Phi_{2 l}(x) P_{2 l}(x) .
\end{aligned}
$$

The radial derivatives in equation (2.27) are computed using second-order accurate finite differences regularized at the origin:

$$
\left.\frac{1}{r} \Phi_{, r}\right|_{i}=\left.2 \Phi_{, r^{2}}\right|_{i}=f_{i} D_{i+1}+f_{i+1} D_{i}
$$

where

$$
\begin{gathered}
D_{i}=2 \frac{\Phi_{i}-\Phi_{i-1}}{r_{i}^{2}-r_{i-1}^{2}}, \quad \Phi_{i} \equiv \Phi\left(r_{i}\right), \\
f_{i}=\frac{r_{i}-r_{i-1}}{\Delta}, \quad \Delta \equiv r_{i+1}-r_{i-1},
\end{gathered}
$$

and

$$
\begin{aligned}
\left.\Phi_{, r r}\right|_{i} & =\left.\left(2 r \Phi_{, r 2}\right)_{, r}\right|_{i} \\
& =g_{i+1} D_{i+1}-g_{i} D_{i},
\end{aligned}
$$

where

$$
g_{i}=\frac{r_{i}+r_{i-1}}{\Delta}
$$

These equations are used at all zones except the origin. The value of $I$ at the origin is found by extrapolation from the neighboring two zones.

\section{REFERENCES}

Binney, J., \& Tremaine, S. 1987, Galactic Dynamics (Princeton: Princeton Univ. Press), chap. 4

Bisnovatyi-Kogan, G. S., \& Zel'dovich, Ya. B. 1970, Astrofizika, 6, 387 (English trans. in Astrophysics, 6, 207)

Freeman, K. C. 1966, MNRAS, 134, 1

Fridman, A. M., \& Polyachenko, V. L. 1984, Physics of Gravitating Systems (New York: Springer), chap. 4

Gottlieb, D., \& Ōrszag, S. A. 197̄7, Numerical Analysis of S̄pectral Methods (Philadephia: SIAM)

Horst, E. 1982, Math. Methods Appl. Sci., 4, 19

Hunter, C. 1975, in Proc. IAU Symp. 69, Dynamics of Stellar Systems, ed. A. Hayli (Dordrecht: Reidel), 195

Lake, G. 1981a, ApJ, 243, 111

.1981b, ApJ, 243,121
Lin, C. C., Mestel, L., \& Shu, F. H. 1965, ApJ, 142, 1431

Penrose, R. 1969, Nuovo Cimento, 1 (special number), 252

Prendergast, K. H., \& Tomer, E. 1970, AJ, 75, 674

Press, W. H., Flannery, B. P., Teukolsky, S. A., \& Vetterling, W. T. 1986, Numerical Recipes: The Art of Scientific Computing (Cambridge: Cambridge Univ. Press)

Rendall, A. D. 1991, Classical and Quantum Gravity, submitted

Shapiro, S. L., \& Teukolsky, S. A. 1987, ApJ, 318, 542

. 1991, Phys. Rev. Lett., 66, 994

Thorne, K. S. 1972, in Magic Without Magic: John Archibald Wheeler, ed. J. Klauder (San Francisco: W. H. Freeman)

Tremaine, S., \& De Zeeuw, T. 1987, in Proc. IAU Symp. 127, Structure and Dynamics of Elliptical Galaxies, ed. P. T. de Zeeuw (Dordrecht : Reidel), 493 Wilson, C. P. 1975, AJ, 80, 175 